\title{
Flow Properties of Polymer Solutions. II. Phenomenological Relation for the Stress Following Sudden Start of Steady Shear Flow*
}

\author{
Kunihiro Osaki, Yoshiyuki EInaga, ${ }^{* *}$ Nobuhiro Yamada, ${ }^{* * *}$ \\ Shin-ichi OHta, ${ }^{* * *}$ and Michio Kurata \\ Institute for Chemical Research, Kyoto University \\ Uji, Kyoto 611, Japan.
}

(Received October 8, 1973)

\begin{abstract}
The stress development at the start of steady shear flow was calculated from experimental data for the strain-dependent relaxation modulus of a concentrated polymer solution with the use of a constitutive equation based on the strain-dependent relaxation spectrum. Calculated results were in close agreement with those observed in the range of relatively low rate of shear, where no stress overshoot at the start of steady shear flow was observed. When stress overshoot was present, the calculated result was in close agreement with the observed up to the time at which the stress reached its maximum value. This time corresponded to a certain value $s_{B}$ of the shear strain, irrespective of the rate of shear, and was accurately predicted from the relaxation modulus using the strain-dependent constitutive model. At longer times the calculated result for the rate of decrease of the stress was too small, compared with the observed, so that the predicted peak of the stress overshoot was too broad. It was concluded that the strain-dependent constitutive model may be applied to polymer solutions when the shear strain is smaller than $s_{\mathrm{B}}$ or when the rate of shear is smaller than $s_{\mathrm{B}} / \tau_{\mathrm{m}}$, where $\tau_{\mathrm{m}}$ is the maximum relaxation time of the polymer solution.
\end{abstract}

KEY WORDS Constitutive Equation / Strain-Dependent Model /

Stress Overshoot / Steady Shear / Relaxation Modulus /

Constitutive equations of a single integral type may be of special importance in describing the nonlinear viscoelasticity of polymer concentrates. ${ }^{1,2}$ Equations of this type can sometimes be derived from molecular theories based on entanglement networks. In the phenomenological sense, they include linear viscoelasticity in a general form as a limiting case and they are very manageable in calculating stresses for any given flow history. Among many possible equations of this type, those based on ratedependent $t^{2,3}$ or strain-dependent ${ }^{2,4}$ relaxation

* Requests for reprints should be addressed to M. Kurata.

** Present address: Department of Polymer Science, Osaka University, Toyonaka.

*** Department of Industrial Chemistry, Kyoto University. Present address: Nippon Tokushu Kento, Ltd., Kisshoin, Minami, Kyoto.

**** Department of Industrial Chemistry, Kyoto University. spectra may be of interest; they may be described in rather general forms without giving specific functional forms for the relaxation spectra and they are very simple in the sense that one value of the memory function may be obtained by specifying only one state (or time) $t^{\prime}$ in the flow history described relative to the state $t$ of observation of stress. It may be noted that equations based on relaxation spectra dependent on some average of the invariant of the strain-rate tensor ${ }^{2,5}$ are more complicated because one value of the memory function is determined by specifying the states over a certain period of time in the flow history.

In previous papers, we have shown that the constitutive equation based on the rate-dependent relaxation spectrum is not suitable for describing the shear relaxation modulus under large strain for a concentrated polymer solution. ${ }^{6,7}$ We have also examined the applicability of the 
equation based on the strain-dependent relaxation spectrum by studying the stress relaxation after application of a double-step shear strain. ${ }^{8}$ It has been shown that the constitutive model based on the strain-dependent spectrum may be capable of describing quantitatively the nonlinear behavior of the polymer solution, unless the strain is very large.

In the present study, we have examined how well the strain-dependent model can derive the shear stress following a sudden start of steady shear flow from the strain-dependent relaxation modulus. It is not to be expected that quantitative comparison is possible over a wide range of rate of shear; the model seems to be incapable of describing stresses in deformations of very large strain or rate of strain. ${ }^{4,8}$ However, the application of the strain-dependent model to the interrelation of the relaxation modulus and the stress development in steady shear flow is still of much interest because it is one of few cases in which two quantities in nonlinear viscoelasticity are related to each other through a fairly general phenomenological equation which does not include any adjustable parameters specific to the material. ${ }^{2,4}$ The result seems to give definite critical values for the strain and the rate of shear up to which the strain-dependent model is applicable.

\section{METHOD}

We deal with the strain-dependent relaxation modulus $G(t, s)$ and the stress development function $\bar{\eta}(t, \kappa)$. The strain-dependent relaxation modulus is the ratio of the shear stress to the shear strain at time $t$ after a sudden application of shear strain $s .^{6}$ The stress development function is the ratio of the shear stress to the rate of shear $\kappa$ at time $t$ after a sudden start of steady shear flow. ${ }^{9}$ According to the constitutive equation based on the strain-dependent relaxation spectrum, one obtains a relation for these quantities, ${ }^{2,4}$

$$
\partial \bar{\eta}(t, \kappa) / \partial t=[\partial s G(t, s) / \partial s]_{s=\kappa t}
$$

This equation implies that the stress development function can be evaluated from the straindependent relaxation modulus.

We examined the applicability of this equation by calculating each term from experimental values of $\bar{\eta}(t, \kappa)$ and $G(t, s)$ for a polymer solution reported previously. ${ }^{7,9}$ The material was a 20-\% solution of polystyrene (sample 14a from Pressure Chemical Company; molecular weight $=$ $1.80 \times 10^{6}$ ) in chlorinated biphenyl (Aroclor 1248 from Monsanto Chemical Company). The experimental results at $30^{\circ} \mathrm{C}$ were used in the calculation.

\section{RESULTS}

Calculated results are given in Figures 1 through 4 , where $\partial \bar{\eta}(t, \kappa) / \partial t$ (thick lines) and $[\partial s G(t, s) / \partial s]_{s=\kappa t}$ (circles) are shown as functions of time $t$. Figure 1 represents the case of relatively low rate of shear at which stress overshoot is not observed, although the nonlinear effect (i.e., the dependence of $\bar{\eta}(t, \kappa)$ on $\kappa$ ) is significant. The results ${ }^{9}$ for $\partial \bar{\eta}(t, \kappa) / \partial t$ or $G(t, s)$ in the limit of $\kappa \rightarrow 0$ or $s \rightarrow 0$, respectively, are shown with a chain line. It is seen that $[\partial s G(t, s) / \partial s]_{s=\kappa t}$ is in close agreement with $\partial \bar{\eta}(t, \kappa) / \partial t$ over the whole range of time scale studied. The result obtained from $G(t ; s)$ may be a little lower in

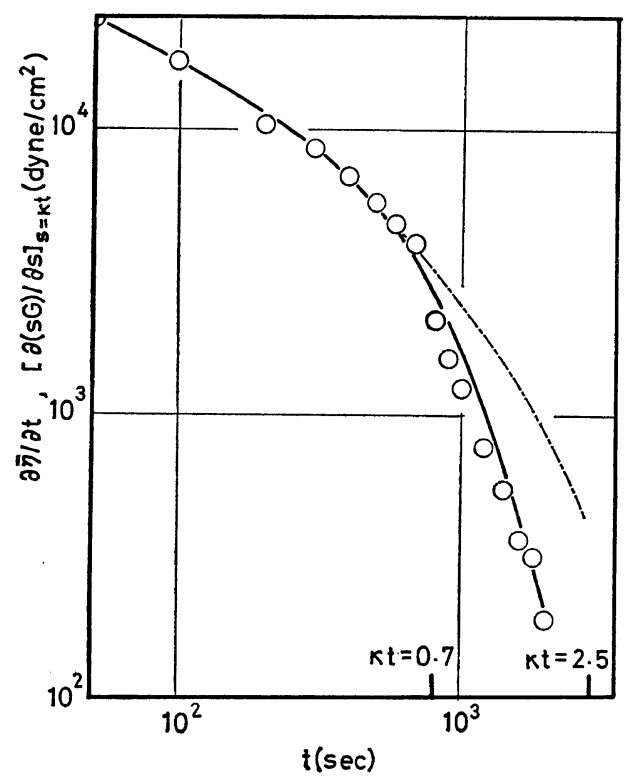

Figure 1. $\partial \bar{\eta} / \partial t$ (solid line) and $[\partial(s G) / \partial s]_{s=\kappa t}$ (circles) plotted against time $t$ for $20-\%$ polystyrene solution in chlorinated biphenyl. Rate of shear $\kappa$ is $8.65 \times 10^{-4} \mathrm{sec}^{-1}$. Chain line represents $\partial \bar{\eta} / \partial t$ or $[\partial(s G) / \partial s]_{s=\kappa t}$ at the limit of $\kappa \rightarrow 0$. 
Flow Properties of Polymer Solutions. II.

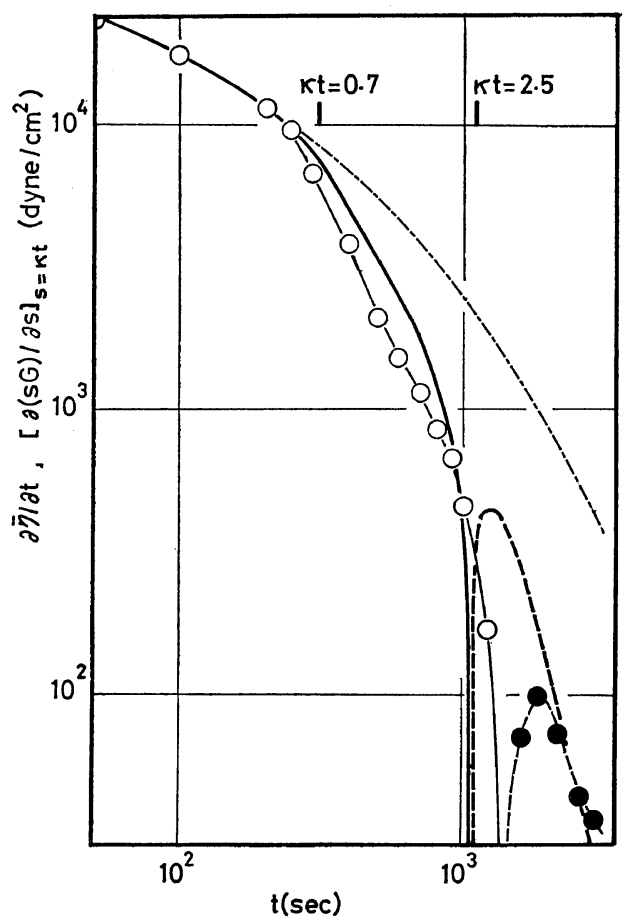

Figure 2. $\partial \bar{\eta} / \partial t$ (heavy lines) and $[\partial(s G) / \partial s]_{s=\kappa t}$ (circles) plotted against time $t$ for $20-\%$ polystyrene solution in chlorinated biphenyl. Rate of shear $\kappa$ is $2.30 \times 10^{-3} \mathrm{sec}^{-1}$. Filled circles and dashed line indicate negative values of $[\partial(s G) / \partial s]_{s=\kappa t}$ and $\partial \bar{\eta} / \partial t$, respectively, and chain line represents $\partial \bar{\eta} / \partial t$ or $[\partial(s G) / \partial s]_{s=\kappa t}$ at the limit of $\kappa \rightarrow 0$.

the long time range but the deviation is not much larger than the error involved in the calculation.

Figures 2 through 4 represent the cases of relatively high rates of shear, at which stress overshoot is observed. In these figures thick dashed lines and filled circles indicate negative values of $\partial \bar{\eta}(t, \kappa) / \partial t$ and $[\partial s G(t, s) / \partial s]_{s=\kappa t}$, respectively. Characteristic features indicated in these figures may be summarized as follows. The agreement between $\partial \bar{\eta}(t, \kappa) / \partial t$ and $[\partial s \boldsymbol{G}(t, s) / \partial t]_{s=\kappa t}$ is satisfactory in the range of relatively short times, i.e., approximately up to the time indicated by $\kappa t=0.7$ in each figure. Then the result obtained from $G(t, s)$ becomes slightly lower than that from $\bar{\eta}(t, \kappa)$ up approximately to the time at which $\partial \bar{\eta}(t, \kappa) / \partial t$ changes sign. In this intermediate range of times, the agreement between circles and thick lines is not very bad,

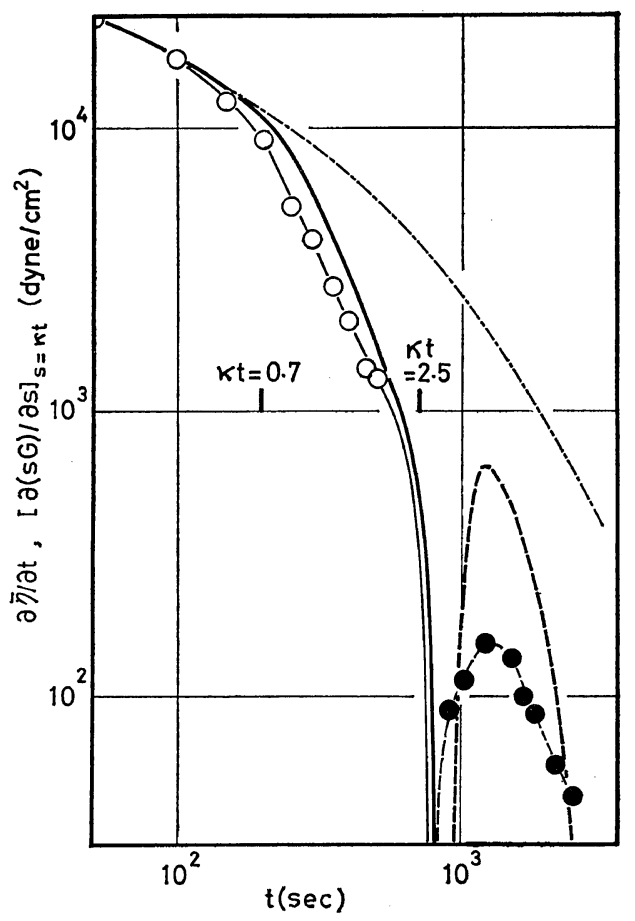

Figure 3. $\partial \bar{\eta} / \partial t$ (heavy lines) and $[\partial(s G) / \partial s]_{s=\kappa t}$ (circles) plotted against time $t$ for $20-\%$ polystyrene solution in chlorinated biphenyl. Rate of shear $\kappa$ is $3.61 \times 10^{-3} \mathrm{sec}^{-1}$. Filled circles and dashed line indicate negative values of $[\partial(s G) / \partial s]_{s=\kappa t}$ and $\partial \bar{\eta} / \partial t$, respectively, and chain line represents $\partial \bar{\eta} / \partial t$ or $[\partial(s G) / \partial s]_{s=\kappa t}$ at the limit of $\kappa \rightarrow 0$.

in view of the large nonlinear effect. It may be noted that the change of sign of $\partial \bar{\eta}(t, \kappa) / \partial t$ occurs at a time very close to that given by $\kappa t=2.5$, for any rate of shear studied. The time at which $[\partial s G(t, s) / \partial s]_{s=\kappa t}$ changes sign is not always in agreement with that for $\partial \bar{\eta}(t, \kappa) / \partial t$ : The peak of $\bar{\eta}(t, \kappa)$, as predicted from $G(t, s)$, appears at too long a time for the low rate of shear. The predicted time for a high rate of shear may be a little too short, but is in fair agreement with the observed. In the range of very long times both $-\partial \bar{\eta}(t, \kappa) / \partial \mathrm{t}$ and $-[\partial s G(t, s) / \partial s]_{s=\kappa t}$ show maxima, but the maximum value of the latter is much lower than that of the former. This result indicates that the peak of $\bar{\eta}(t, \kappa)$, as predicted from $G(t, s)$ with the strain-dependent constitutive model, is broader than the observed result. 


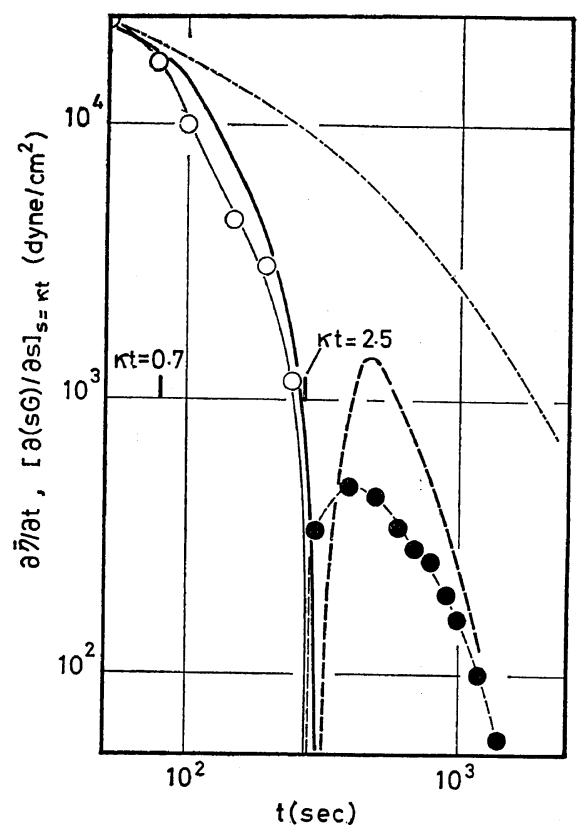

Figure 4. $\partial \vec{\eta} / \partial t$ (heavy lines) and $[\partial(s G) / \partial s]_{s=\kappa t}$ (circles) plotted against time $t$ for $20-\%$ polystyrene solution in chlorinated biphenyl. Rate of shear $\kappa$ is $8.86 \times 10^{-3} \mathrm{sec}^{-1}$. Filled circles and dashed line indicate negative values of $[\partial(s G) / \partial s]_{s=\kappa t}$ and $\partial \bar{\eta} / \partial t$, respectively, and chain line represents $\partial \bar{\eta} / \partial t$ or $[\partial(s G) / \partial s]_{s=\kappa t}$ at the limit of $\kappa \rightarrow 0$.

\section{DISCUSSIONS}

\section{Summary of Results}

It has been pointed out ${ }^{9}$ that the stress development function $\bar{\eta}(t, \kappa)$ is not affected by varying the rate of shear approximately up to the time determined by $\kappa t=0.7$ at any rate of shear studied, i.e., up to a certain strain denoted by $s_{\mathrm{A}}$ for later use. It may also be recalled $^{7}$ that the relaxation modulus is independent of $s$ in the range of $s$ smaller than approximately 0.7 for this polymer solution. Figures 2 through 4 show that the peak of $\bar{\eta}(t, \kappa)$, if any, appears at the time corresponding to a constant strain $s_{\mathrm{B}}$ independent of the rate of shear, where $s_{\mathrm{B}}=2.5$ for the polymer solution studied. The peak is not observed at $\kappa=8.65 \times 10^{-4} \mathrm{sec}^{-1}$, but it is observed at $\kappa=$ $2.30 \times 10^{-3}$. One may tentatively state that the peak does not appear unless it appears within a time of the order of the maximum relaxation time $\tau_{\mathrm{m}}$, approximately $1.5 \times 10^{3} \mathrm{sec}$ in this case, or unless $\kappa$ is high enough to satisfy the relation $s_{\mathrm{B}}<\kappa \tau_{\mathrm{m}}$. The values of $s_{\mathrm{A}}$ and $s_{\mathrm{B}}$ should be independent of temperature. ${ }^{9}$

The qualitative features of the stress development function $\bar{\eta}(t, \kappa)$ are rather well derived from the strain-dependent relaxation modulus in terms of the model based on the strain-dependent relaxation spectrum. Quantitative agreement of the prediction with the observation is attained for a relatively low rate of shear, at which stress overshoot does not appear. The agreement is not very bad even for a higher rate of shear in the range of times satisfying $\kappa t<s_{\mathrm{B}}$ although, precisely speaking, the predicted rate of increase of $\bar{\eta}(t, \kappa)$ is a little too low. The predicted rate of decrease of $\bar{\eta}(t, \kappa)$ following stress overshoot is too low, so that the predicted peak of $\bar{\eta}(t, \kappa)$ is broader than the observed. This discrepancy may be related to the failure of the theory in predicting the overshoot of normal stress for this polymer solution. ${ }^{4}$ The sharp peak would be predicted, as well as the overshoot of the normal stress, if $G(t, s)$ decreased more rapidly than does the observed with increasing strain.

\section{Nature of Nonlinear Viscoelasticity}

According to the results obtained above, one may assume that the degree of nonlinear effect in any time-dependent shear strain is classified in terms of the magnitudes of strain $s$ and rate of shear $\kappa$, as illustrated in Figure 5. This figure represents a map defined by two coordinates $s$ and $\kappa$ so that any time-dependent shear strain represented with a curve or points on the map. (Since we are concerned with the magnitudes of strain or rate of shear, we pay no regard to the sign of $s$ or $\kappa$. In this section $s$ or $\kappa$ should be understood to be absolute values. It may also be remarked that, in describing deformations different from simple shear, one may have to employ invariants of strain and strain rate tensors in the place of $s$ and $\kappa$.) The map is divided into three regions with lines defined by $s=s_{\mathrm{A}}, s=s_{\mathrm{B}}, \kappa=s_{\mathrm{A}} / \tau_{\mathrm{m}}$, and $\kappa=s_{\mathrm{B}} / \tau_{\mathrm{m}}$. These values of $\kappa$ may not be the best choices but we preferred not to increase the number of parameters, since these give the right order of magnitude. 
Flow Properties of Polymer Solutions. II.

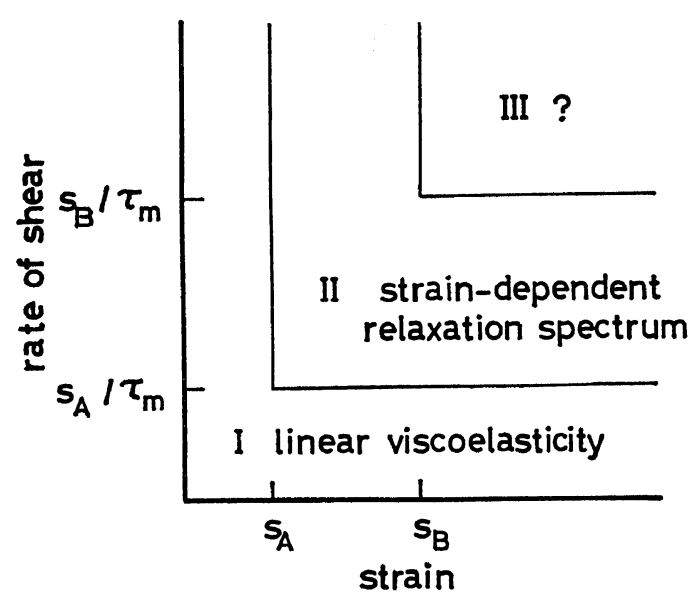

Figure 5. Proposed criterion for applicability of constitutive equation based on the strain-dependent relaxation spectrum. Ordinate and abscissa represent absolute values of rate of shear and shear strain, respectively, in arbitrary scales.

In region $\mathrm{I}$, defined by $s<s_{\mathrm{A}}$ or $\kappa<s_{\mathrm{A}} / \tau_{\mathrm{m}}$ the phenomenological theory of linear viscoelasticity applies; in the neighboring region, defined by $s<s_{\mathrm{B}}$ or $\kappa<s_{\mathrm{B}} / \tau_{\mathrm{m}}$, the constitutive model based on the strain-dependent relaxation spectrum applies. It may be remarked that the constitutive model based on the rate-dependent relaxation spectrum may also be applicable, as argued by many authors, ${ }^{2}$ in the part of region II where the strain is large. In fact, the strain-dependent model gives results similar to those of the ratedependent model for many rheological problems in which $\kappa$ does not vary much and $s$ is large. On the other hand, in region III, defined by $s>s_{\mathrm{B}}$ and $\kappa>s_{\mathrm{B}} / \tau_{\mathrm{m}}$, no constitutive model based on strain-dependent or rate-dependent relaxation spectra is applicable. The constitutive equation effective in this region is so complicated that the specification of one state in the flow history relative to the time of measurement of stress is insufficient to determine a value of the memory function. ${ }^{8}$
The strain-dependent model may seem to be unable to describe the development of normal stress at the start of steady shear flow. In fact, the overshoot of normal stress is not predicted from the relaxation moduli data employed in the present study. ${ }^{4}$ It has also been noted that the rate-dependent model is better than the straindependent model in describing the time at which the normal stress reaches its maximum value..$^{3,4,10}$ However, these results may not necessarily be in conflict with the criterion given above, since the overshoot of normal stress always occurs later than that of shear stress, so that the phenomenon of normal stress overshoot belongs to region III in Figure 5. On the other hand, it is possible that the rate-dependent model is actually effective for solutions of lower concentrations at which the development of normal stress has usually been studied. In any case, it is desirable to compare the development of normal stress and the strain-dependent relaxation modulus at various concentrations.

\section{REFERENCES}

1. A. S. Lodge, "Elastic Liquids," Academic Press, London-New York, N.Y., 1964.

2. M. Yamamoto, "Buttai no Henkeigaku", Seibundo-Shinkosha, Tokyo, 1972.

3. M. Yamamoto, Trans. Soc. Rheol., 15, 331, 783 (1971).

4. M. Yamamoto, Zairyo, 21, 355 (1972).

5. D. C. Bogue, Ind. Eng. Chem., Fundam., 5, 253 (1966).

6. Y. Einaga, K. Osaki, M. Kurata, S. Kimura, and M. Tamura, Polymer J., 2, 550 (1971).

7. Y. Einaga, K. Osaki, M. Kurata, S. Kimura, N. Yamada, and M. Tamura, ibid., 5, 91 (1973).

8. K. Osaki, Y. Einaga, M. Kurata, N. Yamada, and M. Tamura, ibid., 5, 283 (1973).

9. K. Osaki, Y. Einaga, N. Yamada, and M. Kurata, ibid., 6, 72 (1974).

10. M. Sakai, H. Fukaya, and M. Nagasawa, Trans. Soc. Rheol., 16, 635 (1972). 